\title{
ARTHRITIS IN CHILDREN
}

\author{
T R Southwood
}

\section{Diagnosis of juvenile chronic} arthritis

All three of these criteria must be met:

- Arthritis persisting for more than three months

- Onset of arthritis before age 16

- Exclusion of other diseases that may cause arthritis

Arthritis is defined as:

- Swelling of at least one peripheral joint or

- At least two of the following signs-limited range of movement, tenderness or pain on movement of joint, or increased warmth of skin overlying joint

Children with arthritis may not complain of pain, but most do experience some discomfort

Common mistakes in diagnosis of juvenile chronic arthritis are:

Diagnosis in absence of persistent, objective joint swelling

Failure to exclude differential diagnoses
Rheumatic symptoms, such as limping or limb pain, are common in children, yet childhood arthritis and other rheumatic diseases are rare. Diagnosis and treatment of these conditions therefore require a high level of clinical awareness and careful assessment. Laboratory investigations are rarely pathognomonic and are used instead to help differential diagnosis, to detect complications, and to monitor disease activity. Drug treatment depends on a sound knowledge of the variable nature and prognosis of the conditions and a clear understanding of the pharmacology, risks, and benefits of anti-inflammatory and antirheumatic drugs in children.

Persistent swelling of joints characterises a heterogeneous group of diseases termed juvenile chronic arthritis, formerly known as Still's disease. This group of diseases is, for the most part, genetically and clinically distinct from rheumatoid arthritis in adults. About one child in every 1000 has juvenile chronic arthritis, amounting to over 12000 affected children in Britain. Each year one new case would be expected in every 10000 children.

The diagnosis of juvenile chronic arthritis is often difficult initially because swelling of joints may be subtle or absent and complaints of pain may be difficult to elicit from children. Children may present with limping, upper limb dysfunction, torticollis, or non-specific constitutional symptoms such as lethargy, fever, poor appetite, or irritability. Occasionally, features not associated with the musculoskeletal system may predominate: rash, pericarditis, serositis, or organomegaly. Prompt diagnosis and an early start of appropriate treatment are important; the longer the delay, the greater the risk of joint contractures, muscle wasting, and abnormal growth.

\section{Classification of juvenile chronic arthritis}

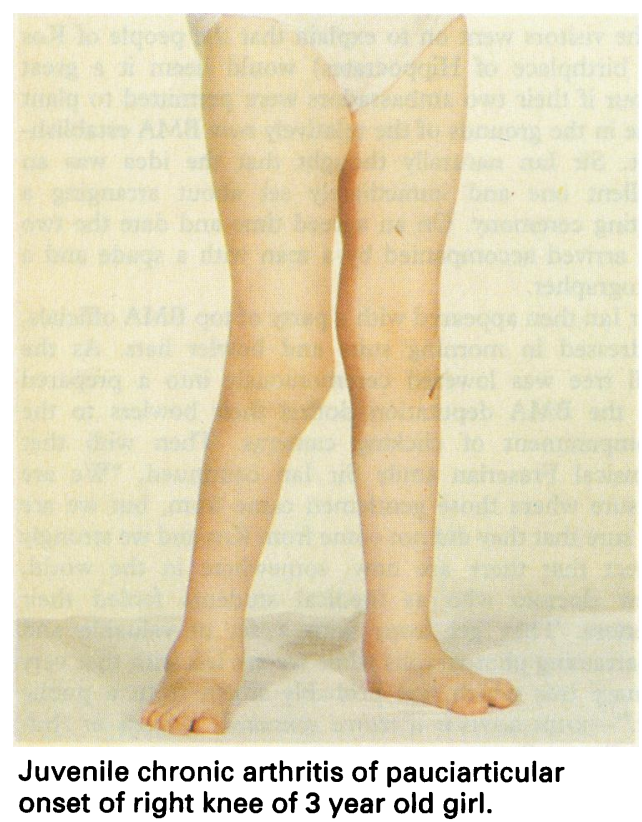

The basis of the classification of juvenile chronic arthritis is the number of joints affected during the first six months of the disease and the presence of extra-articular clinical features. There are three main clinical patterns: pauciarticular onset affects up to four joints, polyarticular onset affects five or more joints, and systemic onset has prominent extra-articular features such as fever and rash.

\section{Fuvenile chronic arthritis of pauciarticular onset}

This is the most common subtype of juvenile chronic arthritis, accounting for over half of cases, and usually affects young (preschool) girls. It is commonly associated with antinuclear antibodies. A fifth of patients develop the clinically silent but potentially blinding complication of chronic anterior uveitis. The articular prognosis for patients is good: in most cases the arthritis is not severe and only persists for a few years, although a third of patients will eventually develop polyarthritis that is difficult to control. 


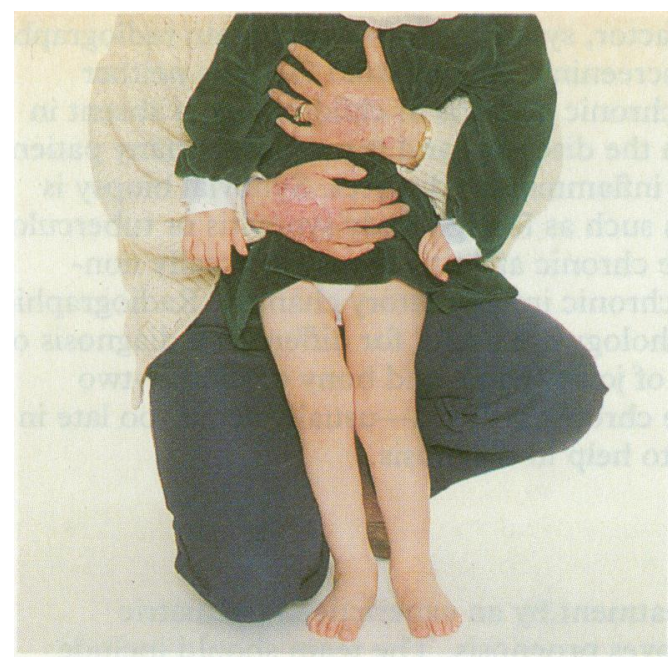

Juvenile chronic arthritis of pauciarticular onset of knee (and dactylitis of a finger) in child whose mother has psoriasis on dorsal surface of her hands.

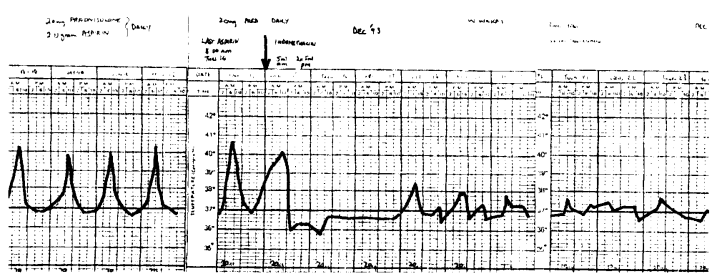

Temperature chart showing characteristic quotidian fever caused by juvenile chronic arthritis of systemic onset. The fever was uncontrolled by prednisolone $20 \mathrm{mg} /$ day and high dose aspirin, but it settled after substitution with indomethacin $20 \mathrm{mg} / \mathrm{kg} / \mathrm{day}$.

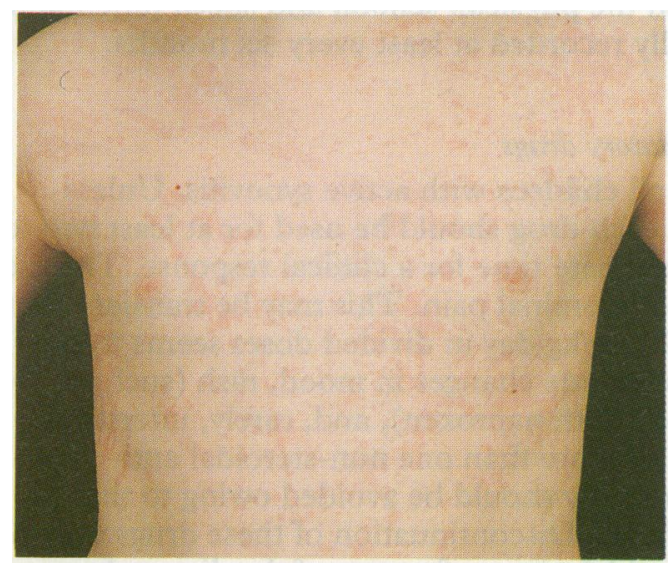

Typical erythematous evanescent rash of juvenile chronic arthritis of systemic onset. Often it is obvious only at the height of fever and is sometimes confined to axillary region and lateral chest wall.

\section{Laboratory investigations}

\section{Key investigations for differential diagnosis of juvenile chronic arthritis}

- Synovial fluid aspiration for microscopy and culture if sepsis is suspected

- Full blood count (and bone marrow aspiration if neoplasia is suspected)

- Measurement of acute phase proteins

- Plain radiographs of affected joint
Two other groups of patients may also present with pauciarticular disease; those with juvenile psoriatic arthritis and those who are predisposed to ankylosing spondylitis. Both of these groups may be difficult to diagnose at the start of disease, as arthritis may predate by many years the characteristic clinical features of psoriasis or of sacroiliitis or spondyloarthritis. Clues to the diagnosis of juvenile psoriatic arthritis without a typical psoriatic rash are a family history of psoriasis, pitting of nails, and an asymmetrical arthritic pattern affecting both small joints (such as those of the finger) and large joints. Juvenile psoriatic arthritis seems to have a poorer prognosis than juvenile chronic arthritis of pauciarticular onset without psoriasis.

Children who are predisposed to ankylosing spondylitis are usually boys who develop a predominantly lower limb arthritis during late childhood or adolescence. An important clinical feature of such children is enthesitis-inflammation and tenderness at the site of insertion of tendons, ligaments, or fascia into bone. The most common clinically detectable sites of enthesitis are around the foot: the insertions of the plantar fascia into the calcaneum, the base of the fifth metatarsal and the metatarsal heads, and the insertion of the Achilles tendon into the calcaneum posteriorally. There is often a family history of "bad backs," inflammatory bowel disease, or acute uveitis. Although radiological evidence of sacroiliitis is rarely present at the start of this disease in children, over half will eventually develop ankylosing spondylitis.

\section{Fuvenile chronic arthritis of polyarticular onset}

This arthritis, which is predominantly symmetrical and affects upper and lower limbs, is found in $30-40 \%$ of children with juvenile chronic arthritis. Most patients are girls who develop arthritis during their preschool years. However, the disease can also start in late childhood or adolescence, and such cases, which account for less than $10 \%$ of cases of juvenile chronic arthritis, are the only ones associated with persistent serological evidence of rheumatoid factor. This probably represents the juvenile manifestation of the adult type of rheumatoid arthritis. Both groups of patients with arthritis of polyarticular onset have a poorer articular prognosis than those with disease of pauciarticular onset; $30-50 \%$ develop bony erosions and active arthritis that persists into adulthood.

\section{Fuvenile chronic arthritis of systemic onset}

This is the rarest form of juvenile chronic arthritis. Most patients develop the disease in early childhood, but patients of any age may be affected, including adults. It is often difficult to diagnose because the earliest features may be extra-articular. These include a characteristic fever; an evanescent, macular, erythematous rash; and evidence of serositis, organomegaly, or lymphadenopathy. The number of arthritic joints varies: about a third of patients develop severe polyarthritis that is resistant to treatment, and the articular outlook for these patients is poor. The life threatening complications of pericarditis and amyloidosis are associated with this disease.

No investigation is pathognomonic of juvenile chronic arthritis, but several are useful for differential diagnosis. Measures of the acute phase response (erythrocyte sedimentation rate, $\mathrm{C}$ reactive protein, and serum viscosity) are raised in about $70 \%$ of patients with juvenile chronic arthritis at diagnosis and are useful for monitoring disease activity. Antinuclear antibodies are found in about half of patients with juvenile chronic arthritis of pauciarticular onset, and their presence correlates with the risk of chronic anterior uveitis. Antinuclear antibodies are not, however, specific for this disease; they are also found in $20 \%$ of patients with polyarticular disease, $5 \%$ of those with systemic disease, patients with many other connective tissue diseases, and $5 \%$ of apparently normal children. 
Screening for rheumatoid factor is not a useful test for childhood arthritis, but it is an important prognostic factor after juvenile chronic arthritis of polyarticular onset has been diagnosed

Tests for rheumatoid factor, synovial biopsy, and plain radiographs are unsuitable for use in screening. Rheumatoid factor is neither specific nor sensitive for chronic arthritis in children-it is absent in over $90 \%$ of patients with the disease-and is present in many patients with infectious and other inflammatory diseases. Synovial biopsy is useful to exclude diseases such as foreign body synovitis or tuberculous arthritis, but with juvenile chronic arthritis biopsy is usually nonspecific and reveals only chronic inflammatory changes. Radiographic features of local bone pathology are useful for differential diagnosis of a swollen joint. Narrowing of joint spaces and bony erosions-two features of severe juvenile chronic arthritis-usually occur too late in the course of the disease to help in diagnosis.

\section{Treatment}

One of the most important factors in successful treatment of juvenile chronic arthritis is the expertise of nurses, physiotherapists, and occupational therapists in paediatric rheumatology

\section{Complications of juvenile chronic arthritis}

Pain and constitutional features

- Including lethargy, anorexia, and irritability Joint contractures

- Pain caused by raised intra-articular pressure and muscle spasm may be contributory

Anaemia

- Usually secondary to chronic inflammation

- May respond to iron supplements or misoprostol for blood loss induced by nonsteroidal anti-inflammatory drugs

Chronic anterior uveitis

- Clinically silent and potentially blinding

- Patients at highest risk have arthritis of pauciarticular onset before age of 6 and are positive for antinuclear antibodies

- Diagnosed by slit lamp examinationscreening every 3-6 months is warranted for all patients with juvenile chronic arthritis except for those with systemic disease, those who are positive for rheumatoid factor, and those who have arthritis and enthesitis

- Treated with topical corticosteroids and mydriatics

Growth disturbance

- Localised-overgrowth (such as leg lengthening due to knee synovitis) or undergrowth (such as premature fusion of finger epiphyses)

- Generalised-growth failure secondary to severe inflammation or treatment with corticosteroids. Treatment with recombinant growth hormone improves growth rate in some cases

Amyloidosis

- Rare-results in proteinuria and

hypoalbuminaemia

- May respond to chlorambucil

Joint failure

- About $5 \%$ of patients with juvenile chronic arthritis eventually require joint replacement - Those at highest risk have polyarticular disease and are positive for rheumatoid factor
Early diagnosis and treatment by an experienced paediatric rheumatology team improves prognosis. The team should include experts in physiotherapy, occupational therapy, social work, nursing, splint making, and psychology. Ready access to orthopaedic surgery, dentistry and orthodontics, ophthalmology, psychiatry, and other paediatric specialties is also important. Treatment usually includes education about the disease, physical therapy, non-steroidal antiinflammatory drugs, and intra-articular corticosteroids.

The progress of the disease must be monitored carefully. Clinical and laboratory evidence of disease activity should be assessed and recorded several times a year. Affected children rarely need hospitalisation, but it is indicated for inability to walk, progressive flexion deformities unresponsive to physiotherapy, and severe extraarticular features of disease (pericarditis, inanition, or anaemia). Physiotherapists, occupational therapists, clinic nurses, teachers, and other therapists should also be closely involved with monitoring disease. In particular, the range of motion of affected joints; the muscle bulk of affected limbs; and the physical, school, and social function of children should be formally recorded at least every six months.

\section{Non-steroidal anti-inflammatory drugs}

These are indicated in all children with active synovitis. Unless adverse effects are noted, each drug should be used for at least two to three months to ensure adequate time for a clinical response. The most common adverse effect is abdominal pain. This may be transient, but if it is not, misoprostal $10-20 \mu \mathrm{g} / \mathrm{kg} /$ day in divided doses seems to be effective. Other problems include changes in mood, rash (such as pseudoporphyria, described with naproxen), and, rarely, interstitial nephritis. Administration of more than one non-steroidal antiinflammatory drug concurrently should be avoided owing to the additive risk of adverse effects. Discontinuation of these drugs may be considered if all clinical and laboratory features of the disease have been quiescent for six months or more.

\section{Corticosteroids}

Intra-articular corticosteroids are indicated for arthritis that is not controlled by non-steroidal anti-inflammatory drugs. Triamcinolone hexacetonide $(0 \cdot 5-1 \mathrm{mg} / \mathrm{kg}$ for each affected joint) is usually administered under sedation or general anaesthesia with aseptic "no touch" techniques. The maximum frequency of intra-articular treatment for individual joints is unknown but can be as often as every six months if there is a beneficial clinical response. Topical or parenteral corticoisteroids may help to treat severe immobility, carditis, or severe anaemia but are otherwise best avoided because of their propensity for retarding growth and other adverse effects. The risk of osteopenia can be minimised with supplemental calcium (0.5-1 $\mathrm{g} /$ day) and vitamin D (calcitriol $0.25 \mathrm{mg} /$ day). Deflazacort may be more "bone sparing" than prednisolone: it is slightly less potent (a dose of $6 \mathrm{mg}$ of deflazacort is equivalent to $5 \mathrm{mg}$ prednisolone) and should be widely available soon. corticosteroids are the main treatment for chronic anterior uveitis. Oral 


\section{Treatment of juvenile chronic arthritis}

Education about the disease

Written and video information appropriate to patient's age

Counselling and social support

Physical therapy and occupational therapy Monitoring and recording of range of movement of joints

Exercises to increase range of movement of joints

Exercises to increase muscle bulk

Hydrotherapy

Splinting-nocturnal resting splints, working splints, dynamic splints

Non-steroidal anti-inflammatory drugs Naproxen $10-20 \mathrm{mg} / \mathrm{kg} /$ day in twice daily doses-suspension available

Ibuprofen $30-60 \mathrm{mg} / \mathrm{kg} / \mathrm{day}$ in 3 or 4 doses daily-suspension available

Piroxicam 0.2-0.3 mg/day once daily-soluble and lingual tablets

Others used in children-indomethacin,

diclofenac, tolmetin, tenoxicam

Aspirin is rarely used

\section{Intra-articular corticosteroids}

Triamcinolone hexacetonide

$1 \mathrm{mg} / \mathrm{kg} /$ joint for knees, hips, and shoulders

$0.5 \mathrm{mg} / \mathrm{kg} / \mathrm{joint}$ for ankles, wrists, and elbows

Methylprednisolone acetate for small joints of hands and tendon sheaths

These drugs are effective for juvenile chronic arthritis of pauciarticular or polyarticular onset Risks-local subcutaneous atrophy, suppression of growth, osteopenia, anaesthesia if given with general anaesthetic

\section{Slow acting antirheumatic drugs}

These drugs are required for persistent active polyarthritis that is not controlled by non-steroidal anti-inflammatory drugs. Methotrexate helps up to $70 \%$ of patients: the starting dose of $0.3 \mathrm{mg} / \mathrm{kg}$ a week (taken orally) is increased at monthly intervals until clinical benefit is noted (up to a maximum dose of $1 \mathrm{mg} / \mathrm{kg}$ a week or $25 \mathrm{mg}$ a week). Intramuscular or subcutaneous injections may improve the absorption of methotrexate in patients with non-responsive disease. Patients should be monitored every month for adverse effects including abnormalities in liver enzymes, bone marrow toxicity, mood changes, alteration of the urinary sediment, and ulceration of the mouth. There is little controlled scientific evidence to support the use of any other antirheumatic drugs in treating juvenile chronic arthritis, although results of open trials indicate that intramuscular gold may be effective. Sulphasalazine $(50-60 \mathrm{mg} / \mathrm{kg}$ daily in divided doses) may also have a role in uncontrolled juvenile chronic arthritis, particularly for patients with arthritis and enthesitis that may be a precursor to ankylosing spondylitis.

Combination chemotherapy (such as methotrexate, intravenous methylprednisolone pulses, and cyclophosphamide) has been advocated for severe recalcitrant juvenile chronic arthritis of systemic onset. Intravenous gammaglobulin (doses of $1-2 \mathrm{~g} / \mathrm{kg}$ ) has also been used for juvenile chronic arthritis of systemic onset, but its efficacy was questionable in a recent controlled trial.

\section{Immunisations}

Very few patients with juvenile chronic arthritis are sufficiently immunosuppressed to warrant avoiding immunisations containing live viruses. Children with moderate to severe juvenile chronic arthritis often experience exacerbations of arthritis after influenza and other viral illnesses, and yearly influenza immunisation should be considered for these high risk children.

\section{Differential diagnosis}

\section{Differential diagnosis of childhood arthritis}

Mechanical or degenerative disorders Avascular necrosis, osteonecrosis, and osteochondritis

Overuse and hypermobility syndromes

Trauma and non-accidental injury*

Inflammatory disorders

Septic arthritis and osteomyelitis*

Reactive arthritis secondary to extra-articular infection

Systemic connective tissue diseases

Juvenile chronic arthritis (diagnosis of exclusion)

Haematological or neoplastic disorders Haemoglobinopathies

Leukaemia, lymphoma, and neuroblastoma* Localised bone or cartilage tumours Bleeding diatheses

Endocrine and metabolic diseases Insulin dependent diabetes mellitus Hypothyroidism and hyperthyroidism

Idiopathic pain syndromes

Localised-algodystrophy, Sudek's atrophy, reflex sympathetic dystrophy

Generalised-fibromyalgia

* Medical emergencies that should be considered for any child presenting with acute joint swelling; any delay in diagnosis can be disastrous.
Many diseases may present with joint symptoms or have predominantly musculoskeletal features.

\section{Mechanical disorders}

Symptoms are usually related to physical activity and are more common in adolescents. They typically worsen during the day whereas inflammatory joint disease tends to be worse on awakening in the morning. The knee, ankle, hip, and back are most often affected, and associated conditions include localised and generalised hypermobility syndromes, developmental abnormalities such as osteochondritis, and structural disorders.

Non-accidental injury-Joint swelling from non-accidental injury may be due to traumatic periostitis, haemorrhage, or epiphyseal fracture.

Any discrepancy between a patient's history and the physical findings should be carefully assessed. A radiographic skeletal survey and bone scan are indicated.

\section{Inflammatory disorders}

Reactive arthritis is one of the commonest causes of acute arthritis in children. It can be defined as an acute arthritis in the absence of intraarticular sepsis that occurs in close temporal association with an extraarticular infection. Rheumatic fever is now seen only rarely, and an isolated post-streptococcal arthritis is more common. Unlike in adults, in children the gastrointestinal tract is the commonest site of extraarticular infection (salmonella, yersinia, campylobacter, or shigella infections). Other forms of reactive arthritis reported in children include Lyme arthritis, mycoplasma arthritis, and illnesses after viral infections (influenza, rubella, coxsackie B, herpesvirus group, mumps, parvovirus B19, hepatitis B, and alphaviruses). Rarely, arthritis may precede other clinical features of infection, such as jaundice, but it more commonly follows the infection by one to three weeks. 


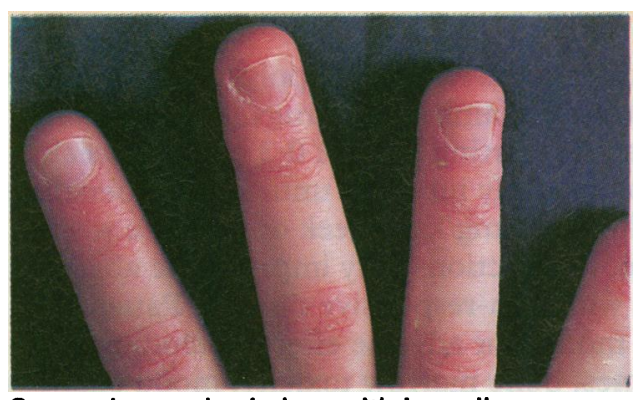

Gottron's papules in boy with juvenile dermatomyositis.

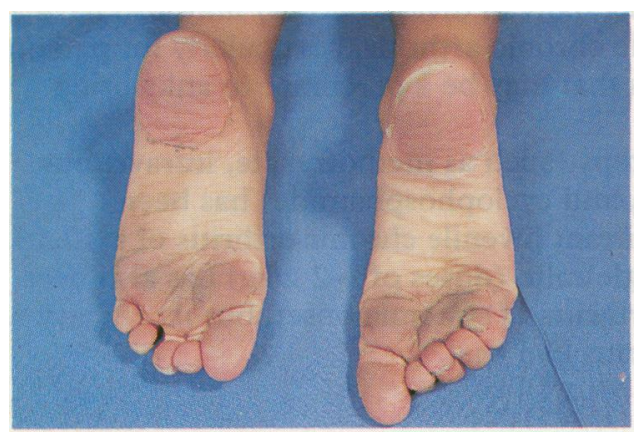

Desquamation of toes and heels of child with Kawasaki disease.

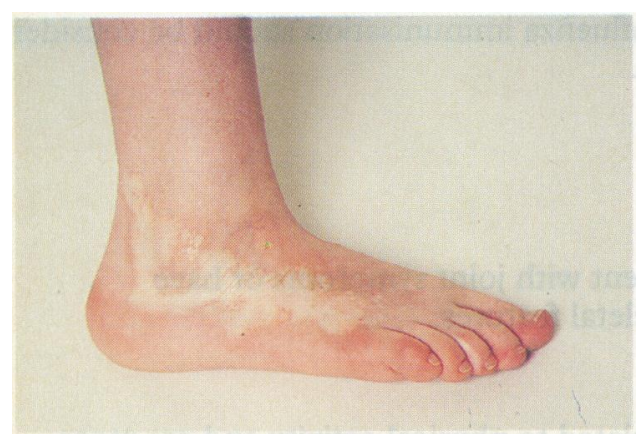

Localised scleroderma of the foot of an 11 year old girl.

\section{Sources of educational material about juvenile chronic arthritis}

- The Children's Chronic Arthritis Association Telephone (01905) 763556

- Young Arthritis Care

Telephone (0171) 9161500

- Arthritis and Rheumatism Council

Telephone (01246) 558033

Handbooks available:

When a young person has arthritis: a guide for teachers

When your child has arthritis: a handbook for parents

- Academy Television

Telephone (0113) 461528

Kids like us-A disease education video for patients with juvenile chronic arthritis

T R Southwood is senior lecturer in paediatric rheumatology in the department of rheumatology, University of Birmingham.

The ABC of Rheumatology is edited by Michael L Snaith, senior lecturer in rheumatology at Nether Edge Hospital, Sheffield.
Septic arthritis-Children with septic arthritis usually seem very unwell with high fever, a hot swollen joint, and severely restricted range of joint movement. Affected joints must be aspirated for diagnostic purposes before treatment is started. Patients should be treated with intravenous antibiotics for two to three weeks, followed by oral antibiotics until the erythrocyte sedimentation rate is normal and there are no clinical findings.

Systemic lupus erythematosus is rare in children. Unlike in adults, prominent presenting features in children include growth failure, constitutional disorders (fever, poor weight gain), and nephritis. Very rarely, transplacental transmission of maternal autoantibodies may result in a neonatal lupus syndrome characterised by congenital complete heart block, dermatitis, and cytopenia. In general, paediatric systemic lupus erythematosus is most severe during the first year after onset. The 15 year survival for children treated at major centres is $85-90 \%$.

Fuvenile dermatomyositis can be diagnosed by the combination of a progressive proximal myopathy and the typical dermatitis. Unlike adult dermatomyositis, it is not associated with an increased prevalence of neoplasia. Arthritis occurs in a third of cases. Life threatening complications include respiratory failure and aspiration. Joint contractures and soft tissue calcinosis may complicate the course of the disease. Concentrations of muscle enzymes (creatine phosphokinase, aspartate aminotransferase, lactate dehydrogenase) are commonly elevated, and serial monitoring is a valuable guide to disease activity. Electromyography and muscle biopsy should be reserved for the diagnosis of atypical cases. Muscle oedema can be quantified using magnetic resonance imaging, and this may be useful for monitoring and for guiding muscle biopsy.

Henoch-Schoenlein purpura - The combination of large joint arthritis, abdominal pain, and a purpuric rash over the lower legs and buttocks is sufficient to diagnose Henoch-Schoenlein purpura. It is the commonest vasculitis in childhood. The disease is usually transient, but about $3 \%$ of sufferers develop important nephritis and it is one of the commoner causes of chronic renal failure in children. Rarely, recurrent episodes of arthritis may occur.

Kawasaki disease is the second most common vasculitis in childhood and is probably underdiagnosed in Britain. The presence of at least five of six criteria is required for the diagnosis: prolonged spiking fever $(>5$ days), oral mucositis, polymorphous rash, cervical lymphadenopathy, conjunctivitis, and oedema followed by peeling of the extremities. Early diagnosis and treatment with intravenous gammaglobulin have substantially reduced the complication of coronary artery aneurysms. Arthritis is usually a transient feature of Kawasaki disease.

Scleroderma - Unlike in adults, scleroderma in children is usually localised. It may be associated with arthritis as part of a mixed connective tissue disease.

\section{Neoplasia}

Arthritis may result from leukaemia, neuroblastoma, lymphoma, and, less often, from localised bone or joint related tumours. Very rarely, children with musculoskeletal symptoms may have a "preleukaemic" condition, the diagnosis only being confirmed months after the onset of symptoms. The commonest localised bony tumour of children is osteoid osteoma. Others include osteochondroma, chondroma, fibrous cortical defects, bone cysts, and eosinophilic granuloma. Malignant bone tumours such as Ewing's sarcoma and osteosarcoma are rare in children.

\section{Idiopathic or stress associated conditions}

The most dramatic musculoskeletal pains in young people are often associated with conditions which are not clearly inflammatory or mechanical. Emotional stress (overt or covert) is common in these patients and may arise from learning difficulties, overachievement, or non-accidental trauma. Associated features include easy fatiguability, poor attendance at school, pronounced limb or bodily dysfunction, and a non-restorative sleep pattern characterised by fatigue on awakening. 